\title{
Extracting audio signals from 4-track analogue tape and digital standalone multitrack machine material as a part of preservation
}

\begin{abstract}
In facing ever-growing technology of audio peripherals, there is a concern of balancing in delivering technical knowledge to students dealing with audio recordings in the Music Department at Putra University, Malaysia (UPM). It seems to be difficult for them to do current multitrack recordings and at the same time maintaining the aesthetic aspect of past technology. In other words, if one preserves the recorded item, they have also to care about the specific features coming with these recordings. It is not the primary recorded subject alone they may have to consider. There is an urgency to migrate the recorded material such as cassette tapes that come in varying forms ranging from Ferric-Oxide, to Cro2, and Metal. These carriers are warranted to be extracted and migrate onto a more stable medium, not only in general as everywhere in the world, but also since it has become the university policy that equipment's exceeding certain years will be disposed of. The selection focuses on the recording materials that includes live musical performances, multitrack musical compositions, and final recording projects. In this context the 'recording' may just mean the audio information. It is important for any archive to establish a general policy with regard to the limits of its collection as was pointed out repeatedly (Schuursma, 2010). Viewing from the perspective of preservation (Musib, 2015) of content, a lack of awareness in handling these carriers may lead to complications when extracting the signal from still available machines. The paper is intended to discuss the challenges and requirements in the process of signal extractions using exemplarily the two formats, namely the 4-track recorder and the digital standalone multitrack machine.
\end{abstract}

Keyword: Signal extraction; 4-track cassette recorder; Digital standalone multitrack recorder; Cassette types; Sound aesthetics 